\title{
CANCELLATION IN THE STONE-ČECH COMPACTIFICATION OF A DISCRETE SEMIGROUP
}

\author{
by NEIL HINDMAN* and DONA STRAUSS
}

(Received 1st September 1992)

\begin{abstract}
We investigate both left and right cancellation in the Stone-Cech compactification $\beta S$ of a discrete semigroup $S$, obtaining several results for arbitrary semigroups $S$ and others for more restricted semigroups. In particular, if $S$ is the semigroup of injective functions from a set to itself we determine precisely which pairs $x, y$ and $S$ have some $p \in \beta S$ with $p x=p y$. We also obtain several new results about right cancellation in $(\beta N,+)$.
\end{abstract}

1990 Mathematics Subject Classification: 22A15.

\section{Introduction}

Given a discrete semigroup $(S, \cdot)$ it is well known that one can define an extension of $\cdot$ to $\beta S$, the Stone-Čech compactification of $S$, so that $(\beta S, \cdot)$ is a left topological semigroup with $S$ contained in its topological centre. (By "left topological" we mean that for each $p \in \beta S$ the function $\lambda_{p}$ defined by $\lambda_{p}(q)=p \cdot q$ is continuous. The "topological centre" is $\left\{p \in \beta S: \rho_{p}\right.$ is continuous $\}$ where $\left.\rho_{p}(q)=q \cdot p\right)$.

The algebraic structure of $\beta S$ is of intrinsic interest since $\beta S$ is the $R M C$ compactification of $S$ [3]. In addition, the algebraic structure has been often useful in obtaining results in Ramsey Theory. See for example the survey [13]. We are concerned in this paper with cancellation in $\beta S$.

In [10] a characterization was obtained of the points $p$ of $\beta S$ at which left cancellation holds. (It is condition (b) of Theorem 2.2 below.) Using this characterization one was then able to show that there are points in the closure of the smallest ideal of $(\beta \mathbb{N},+)$ at which left cancellation holds (while it must fail at all points of the smallest ideal itself). Specializing to the semigroup $(\beta \mathbb{N},+)$, in [4] a long list of conditions equivalent to left cancellation holding at $p$ were obtained.

In this paper we extend these results in two directions. In Section 2, we deal with extending the list of conditions characterizing left cancellation in some more or less arbitrary semigroups. In the process we are led to investigate which points $x$ and $y$ of a left cancellative semigroup $S$ must have some $p \in \beta S$ with $p x=p y$, completely solving this problem in the case $S$ is the semigroup of injective functions from a set to itself. In

*This author gratefully acknowledges support received from the National Science Foundation via grant DMS 90-25025. 
Section 3 we deal with the question of when " $p \notin p \cdot(\beta S \backslash S)$ " can be added to the list of characterizations.

The general results obtained, as well as all the earlier special results about $(\beta \mathbb{N},+)$, deal with left cancellation. The reason for this is the choice of continuity which we have made for the operation in $\beta S$. Had we chosen to make $\rho_{p}$ continuous for each $p \in \beta S$, the results would speak about right cancellation. The problem of cancellation on the side opposite to the continuity seems to be a significantly more difficult problem.

At this point, a word is probably in order about the reasons for our choice of continuity, which is the customary choice for the first author while the choice of right continuity is the customary choice of the second author. The reason for this choice is that our examples involving the semigroup of injective functions naturally utilize a left cancellative semigroup.

In Section 4 we address the problem of right cancellation, obtaining several results for the semigroup $(\beta \mathbb{N},+)$. Included here are examples of the interaction between left and right cancellation. In Section 5 we apply results involving homomorphic images of $\beta \mathbb{N}$, obtaining in particular some necessary conditions for right cancellation.

We take the points of $\beta S$ to be the ultrafilters on $S$, identifying the points of $S$ with the principal ultrafilters on $S$. Given $A \subseteq S, \bar{A}=c l A=\{p \in \beta S: A \in p\}$. The set $\{\bar{A}: A \subseteq S\}$ is a basis for the open sets of $\beta S$. In the semigroup $(S,)^{\circ}$, given $A \subseteq S$ and $x \in S$, one has $A / x=\{y \in S: y \cdot x \in A\}$. Then given $p, q \in S$ one has $A \in p \cdot q \Leftrightarrow\{x \in S: A / x \in p\} \in q$. (If the operation is written + we write $A-x=\{y \in S: y+x \in A\}$ and have $A \in p+q \Leftrightarrow$ $\{x \in S: A-x \in p\} \in q$ ). See [12] for an elementary derivation of these facts. We take $\mathbb{N}=\{1,2,3, \ldots\}$, writing $\omega$ for $\mathbb{N} \cup\{0\}$.

We close this introduction with a well known lemma which is very useful in our discussion of right cancellation. Recall that an $F$-space is a completely regular Hausdorff space in which disjoint co-zero sets are completely separated. In case $X$ is compact, this is equivalent to the assertion that disjoint co-zero sets have disjoint closures, i.e. that $X$ is an $F^{\prime}$-space. It is well known that if $X$ is discrete, then both $\beta X$ and $X^{*}=\beta X \backslash X$ are $F$-spaces. (See $[8]$ for these facts and unfamiliar terminology.)

Lemma 1.1. Let $X$ be a compact Hausdorff space. Then $X$ is an F-space if and only if whenever $A$ and $B$ are $\sigma$-compact subsets of $X$ such that $A \cap(\mathrm{cl} B)=(\mathrm{cl} A) \cap B=\varnothing$, one has $(\mathrm{cl} A) \cap(\mathrm{cl} B)=\varnothing$.

Proof. For the sufficiency simply observe that co-zero sts are $\sigma$-compact. For the necessity, write $A=\bigcup_{n=1}^{\infty} A_{n}$ and $B=\bigcup_{n=1}^{\infty} B_{n}$ where each $A_{n}$ and each $B_{n}$ is compact. Given $n, A_{n}$ and $c l B$ are disjoint compact sets so pick disjoint co-zero sets $U_{n}$ and $V_{n}$ with $A_{n} \subseteq U_{n}$ and $c l B \subseteq V_{n}$. Likewise pick disjoint co-zero sets $U_{n}^{\prime}$ and $V_{n}^{\prime}$ with $c l A \subseteq U_{n}^{\prime}$ and $B_{n} \subseteq V_{n}^{\prime}$. For each $n$, let $G_{n}=U_{n} \cap \bigcap_{k=1}^{n} U_{k}^{\prime}$ and $H_{n}=V_{n}^{\prime} \cap \bigcap_{k=1}^{n} V_{k}$. Then for all $n$ and $m G_{n} \cap H_{m}=\varnothing, A_{n} \subseteq G_{n}$, and $B_{m} \subseteq H_{m}$. Thus $A \subseteq \bigcup_{n=1}^{\infty} G_{n}$ and $B \subseteq \bigcup_{n=1}^{\infty} H_{n}$. Since $\bigcup_{n=1}^{\infty} G_{n}$ and $\bigcup_{n=1}^{\infty} H_{n}$ are disjoint co-zero sets, they have disjoint closures.

\section{Left cancellation in $\beta S$ and separating points of $S$}

We begin by stating from [4], [10] and [16] a long list of conditions characterizing 
left cancellation in $(\beta \mathbb{N},+)$. Recall that two members of $\beta \mathbb{N}$ are type equivalent $(p \approx q)$ if and only if there is a permutation $f$ of $\mathbb{N}$ such that $f^{\beta}(p)=q$, while $p$ precedes $q$ (in the Rudin-Kiesler order) provided there is a function $f: \mathbb{N} \rightarrow \mathbb{N}$ with $f^{\beta}(q)=p$. We write $p \leqq q$ if $p$ precedes $q$ and write $p<q$ if $p \leqq q$ and $p \not q$.

Theorem 2.1. Let $p \in \mathbb{N}^{*}$. The following statements are equivalent.

(a) $\lambda_{p}$ is one-to-one on $\beta \mathbb{N}$ (i.e. left cancellation holds at $p$ );

(b) for each $A \subseteq \mathbb{N}$, there exists $B \subseteq \mathbb{N}$ such that $A=\{x \in \mathbb{N}: B-x \in p\}$;

(c) $\{p+n: n \in \mathbb{N}\}$ is discrete;

(d) for each $q \in \mathbb{N}^{*}, p<p+q$;

(e) for each $q \in \mathbb{N}^{*}, p \neq p+q$;

(f) $p \notin p+\beta \mathbb{N}$;

(g) $p \notin p+\mathbb{N}^{*}$;

(h) there is an increasing sequence $\left\langle x_{n}\right\rangle_{n=1}^{\infty}$ in $\mathbb{N}$ such that for each $k \in \mathbb{N},\left\{x_{n}: x_{n+1}\right\rangle$ $\left.x_{n}+k\right\} \in p$.

(i) there is a one-to-one function $f: \mathbb{N} \rightarrow \beta \mathbb{N}$ such that $\{f(n): n \in \mathbb{N}\}$ is discrete and for all $q \in \beta \mathbb{N}, f^{\beta}(q)=p+q$;

(j) there is a function $g: \mathbb{N} \rightarrow \mathbb{N}$ such that for all $q \in \beta \mathbb{N}, g^{\beta}(p+q)=q$;

(k) there is a function $h: \mathbb{N} \rightarrow \mathbb{N}$ such that for all $q \in \beta \mathbb{N}, h^{\beta}(p+q)=p$.

Proof. [4, Theorem 2.1], [10, Theorem 4.2], [16, Theorem 2] and the observation that for $n \in \mathbb{N}, p \neq p+n$ so that $(g)$ implies $(f)$.

We restrict to $p \in \mathbb{N}^{*}$ above since for $p \in \mathbb{N}, \lambda_{p}$ is always one-to-one [11, Lemma 2.4].

We now extend portions of Theorem 2.1 to a more general setting. Recall that a subset $D$ of a topological space $X$ is strongly discrete if and only if there is a collection $\left\langle U_{x}\right\rangle_{x \in D}$ of open subsets of $X$ with each $x \in U_{x}$ and with $U_{x} \cap U_{y}=\varnothing$ whenever $x \neq y$.

Theorem 2.2. Let $(S,)^{\prime}$ be a discrete semigroup and let $p \in \beta S$. Statement (a) implies statements (b), (c) and (d), which are equivalent. These statements imply statements (e) and (f) which are equivalent. If $S$ is countable, all statements are equivalent;

(a) $\lambda_{\rho}$ is one-to-one on $S$ and $\{p \cdot x: x \in S\}$ is strongly discrete;

(b) for each subset $A$ of $S$ there exists $B \subseteq S$ such that $A=\{x \in S: B / x \in p\}$;

(c) $\lambda_{p}$ is one-to-one on $\beta S$;

(d) $\lambda_{p}$ is one-to-one on $\beta S$ and $\{p \cdot x: x \in S\}$ is discrete;

(e) $\lambda_{p}$ is one-to-one on $S$ and $\{p \cdot x: x \in S\}$ is discrete;

(f) for each $x \in S$ and each $q \in \beta S \backslash\{x\}, p \cdot x \neq p \cdot q$.

Proof. To see that (a) implies (b), pick for each $x \in S$ some $C_{x} \in p \cdot x$ such that $\bar{C}_{x} \cap \bar{C}_{y}=\varnothing$ whenever $x \neq y$. Let $A \subseteq S$ be given and let $B=\bigcup_{x \in A} C_{x}$. To see that $A=\{x \in S: B / x \in p\}$, let $x \in A$. Then $C_{x} \subseteq B$ so $B \in p \cdot x$ so $B / x \in p$. Now let $x \in S$ be given with $B / x \in p$, since also $\left(C_{x}\right) / x \in p$, pick $a \in(B / x) \cap\left(C_{x}\right) / x$. Then $a x \in B \cap C_{x}$. Since $a x \in B$, pick $y \in A$ with $a x \in C_{y}$. Since $C_{y} \cap C_{x} \neq \varnothing$, we have $y \neq x$ so $x \in A$. 
Then (b) and (c) are equivalent is [10, Theorem 4.2(b)]. (The fact that (b) implies (c) is routine: Let $q$ and $r$ be distinct members of $\beta S$ and pick $A \in q \backslash r$. Pick $B$ such that $A=\{x \in S: B / x \in p\}$. Then $B \in(p \cdot q) \backslash(p \cdot r)$.)

That (d) implies (c) is trivial. To see that (c) implies (d), let $x \in S$ and suppose $p \cdot x \in c l\{p \cdot y: y \in S \backslash\{x\}\}$. Since $c l\{p \cdot y: y \in S \backslash\{x\}\}=p \cdot(\beta S \backslash\{x\})$ by the continuity of $\lambda_{p}$ we have $p \cdot x=p \cdot q$ for some $q \in \beta S \backslash\{x\}$, a contradiction.

That (d) implies (e) is trivial. The proof that (f) implies (e) is identical to the proof that (c) implies (d). To see that (e) implies (f) one simply again utilizes the fact that $c l\{p \cdot y: y \in S \backslash\{x\}\}=p \cdot(\beta S \backslash\{x\})$.

Finally it is well known (and an easy exercise) that a countable subset of a regular space is discrete if and only if it is strongly discrete. Consequently if $S$ is countable we have (e) implies (a).

Statements (a) and (e) of Theorem 2.2 lead us to ask when we can guarantee that $\lambda_{p}$ is one-to-one on $S$. It is not hard to see that this must always be true if $S$ is cancellative. (It is also a consequence of Theorem 2.5 below.) It is perhaps surprising that left cancellation is not sufficient.

Lemma 2.3. Let $S$ be a semigroup and let $x, y \in S$. There exists $p$ in $\beta S$ such that $p \cdot x=p \cdot y$ if and only if whenever $F$ is a finite partition of $S$ there is some $A \in F$ with $A \cdot x \cap A \cdot y \neq \varnothing$. More generally, given any discrete set $X$ and any functions $f$ and $g$ from $X$ to $X$; there is some $p \in \beta X$ with $f^{\beta}(p)=g^{\beta}(p)$ if and only if given any finite partition of $X$ there is some $A \in F$ with $f[A] \cap g[A] \neq \varnothing$.

Proof. The first statement is the special case of the second where $S=X, f=\rho_{x}$, and $g=\rho_{y}$ so we prove the second statement. Given $A \in p$ one has $f[A] \in f^{\beta}(p)$ and $g[A] \in g^{\beta}(p)$ so the necessity is immediate. For the sufficiency assume that for each $p \in \beta X, f^{\beta}(p) \neq g^{\beta}(p)$ and pick $U_{p}$ and $V_{p}$, disjoint neighbourhoods of $f^{\beta}(p)$ and $g^{\beta}(p)$ respectively. Pick $A_{p} \in p$ with $f^{\beta}\left[\bar{A}_{p}\right] \subseteq U_{p}$ and $g^{\beta}\left[\bar{A}_{p}\right] \subseteq V_{p}$. Then $\left\{\bar{A}_{p}: p \in \beta X\right\}$ is an open cover of $\beta X$ so pick $p(1), p(2), \ldots, p(n)$ with $X=\bigcup_{i=1}^{n} A_{p(i)}$. Let $B_{1}=A_{p(1)}$ and for $i \in\{2,3, \ldots, n\}$, let $B_{i}=A_{p(i)} \backslash \bigcup_{j=1}^{i-1} A_{p(j)}$. Then the partition $\left\{B_{1}, B_{2}, \ldots, B_{n}\right\}$ of $X$ has $f\left[B_{i}\right] \cap g\left[B_{i}\right]=\varnothing$ for each $i \in\{1,2, \ldots, r\}$.

Corollary 2.4. Let $X$ be discrete and let $f: X \rightarrow X$ with no fixed points. Then $f^{\beta}: \beta X \rightarrow \beta X$ has no fixed points. More generally, if $\varnothing \neq D \subseteq X$ and $f: D \rightarrow X$ with no fixed points then $f^{\beta}: \beta D \rightarrow \beta X$ has no fixed points.

Proof. The second statement follows from the first since $f$ may be extended to all of $X$ by sending $X \backslash D$ to any point of $D$. To see the first statement observe that by [6, Lemma 9.1] there exists a three cell partition $F$ of $X$ with $A \cap f[A]=\varnothing$ for each $A \in F$. The result follows from Lemma 2.3 by taking $g$ to be the identity.

Observe that of course if one hopes to have $p \cdot x \neq p \cdot y$ whenever $x$ and $y$ are distinct members of $S$ and $p \in \beta S$, one must have that $S$ is left cancellative. As a consequence of 
the following theorem, one does obtain that conclusion if $S$ is cancellative. (We add the left-right switch to the theorem since it causes us no additional work.)

Theorem 2.5. Let $(S, \cdot)$ be a semigroup and let $x$ and $y$ be distinct members of $S$.

(1) Assume that for all $s, t \in S$, if $s x \neq s y, t x \neq t y$, and $s x=t x$, then $s y=t y$. Let $p \in \beta S$. Then $p \cdot x=p \cdot y$ if and only if $p \in c l\{s \in S: s x=s y\}$.

(2) Assume that for all $s, t \in S$, if $x s \neq y s, x t \neq y t$, and $x s=x t$, then $y s=y t$. Let $p \in \beta S$. Then $x \cdot p=y \cdot p$ if and only if $p \in c l\{s \in S: x s=y s\}$.

Proof. (1) Let $E=\{s \in S: s x=s y\}$. The sufficiency is immediate. (Since $\rho_{x}$ and $\rho_{y}$ agree on $E$, they agree on $c l E$.) If $E=S$, the necessity is also trivial so we assume $E \neq S$ and pick $a \in S \backslash E$. Define $f: S \rightarrow S$ as follows. For $s \in S \backslash E$, let $f(s x)=s y$. (Our hypothesis guarantees that this makes sense.) For $t \in S$ with $t \notin(S \backslash E) \cdot x$, let $f(t)=a x$. Then for all $t \in S, f(t) \neq t$. Suppose $p \notin c l E$ and pick a net $\left\langle s_{\alpha}\right\rangle_{\alpha \in I}$ in $S \backslash E$ which converges to $p$. Then for each $\alpha \in I f\left(s_{\alpha} x\right)=s_{\alpha} y$. Since $f^{\beta}$ and $\rho_{x}$ and $\rho_{y}$ are continuous we have $f^{\beta}\left(s_{\alpha} x\right)$ converges to $f^{\beta}(p \cdot x)$ while $s_{a} y$ converges to $p \cdot y$. That is $f^{\beta}(p \cdot x)=p \cdot y$. By Corollary $2.4, p \cdot x \neq f^{\beta}(p \cdot x)=p \cdot y$, a contradiction.

The proof of (2) is identical, using the continuity of $\lambda_{x}$ and $\lambda_{y}$.

We only state the corollary corresponding to (1).

Corollary 2.6. Let $(S, \cdot)$ be a semigroup and let $x$ and $y$ be distinct members of $S$. Assume that $s x \neq s y$ for all $s \in S$ and that for all $s$ and $t$ in $S$, if $s x=t x$ then $s y=t y$. Then for all $p \in \beta S, p \cdot x \neq p \cdot y$.

Proof. $\{s \in S: s x=s y\}=\varnothing$.

We shall see later that left cancellation is not sufficient to guarantee that $\lambda_{p}$ is one-to-one on $S$ for all $p \in \beta S$. We set out to show, in Theorem 2.8 , that a left cancellative semigroup cannot be completely bad in this regard.

We feel that the next result, which is for us a lemma for Theorem 2.8 , is interesting in jts own right.

Theorem 2.7. Let $S$ be a left cancellative semigroup and let $x \in S$ such that $x$ is not $a$ left identity for $S$. Then for all $p \in \beta S, p \cdot x \neq p$ and $p \cdot x^{2} \neq p \cdot x$.

Proof. Observe that the second conclusion follows from the first (since $p \cdot x \in \beta S$ ). For all $x \in S, \rho_{x}(s) \neq s$. (For if $s x=s$, then for all $t \in S, s x t=s t$ and hence $x t=t$.) Again invoking [6, Lemma 9.1] we have some $A_{0}, A_{1}, A_{2}$ such that $S=\bigcup_{i=0}^{2} A_{i}$ and $A_{i} \bigcap$ $\rho_{x}\left[A_{i}\right]=\varnothing$ for $i \in\{0,1,2\}$. Let $p \in \beta S$ be given and pick $i \in\{0,1,2\}$ with $A_{i} \in p$. Then $\rho_{x}\left[A_{i}\right] \in \rho_{x}(p)$ so $p \neq p \cdot x$. 
Theorem 2.8. Let $S$ be a left cancellative semigroup. Then there exist $x$ and $y$ in $S$ such that for all $p \in \beta S, p \cdot x \neq p \cdot y$.

Proof. Assume first that some $x \in S$ is not a left identity for $S$. Then by Theorem 2.7 one has for all $p \in \beta S, p \cdot x \neq p \cdot x^{2}$. Thus we may assume every element of $S$ is a left identity, so that $S$ is a "right zero" semigroup. Then given any $x \in S \rho_{x}$ is constantly equal to $x$ on $S$ and hence on $\beta S$, since $\rho_{x}$ is continuous and $S$ is dense in $\beta S$. Thus for all $p \in \beta S$ and all $x \in S, p \cdot x=x$ so if $x \neq y$ and $p \in \beta S, p \cdot x \neq p \cdot y$.

We are concerned, given a left cancellative semigroup $S$, with determining which points $x$ and $y$ in $S$ can be separated by each $\lambda_{p}$. We solve this question completely for a special class of semigroups-the semigroup of injective functions from a set to itself. These semigroups hold a special place among left cancellative semigroups since they contain copies of all civilized left cancellative semigroups. (Here "civilized" means $\lambda_{x} \neq \lambda_{y}$ whenever $x \neq y$. That is, given $x \neq y$ some $c$ has $x c \neq y c$. In this case the function $\phi: S \rightarrow\{f: f: S \stackrel{1-1}{\longrightarrow} S\}$ defined by $\phi(x)=\lambda_{x}$ is one-to-one and a homomorphism.)

Theorem 2.9. Let $A$ be $a$ set and let $S=\{f: f: A \stackrel{1-1}{\longrightarrow} A\}$. Let $g, h \in S$ and let $E=\{a \in A: g(a)=h(a)\}$. Define $\theta: g[A \backslash E] \rightarrow h[A \backslash E]$ by $\theta(g(a))=h(a)$ for $a \in A \backslash E$. There is some $p \in \beta S$ with $p g=p h$ if and only if for some $n \in \mathbb{N}, \theta^{n}=\varnothing$.

Proof. Sufficiency. If $g=h$ the conclusion is trivial so we assume $g \neq h$. We claim first that $A$ must be infinite in this case. Suppose instead $A$ is finite. Then $g$ is onto $A$. We show that range $\theta \subseteq g[A \backslash E]$, so that for all $n$, domain $\theta^{n}=g[A \backslash E] \neq \varnothing$, a contradiction. To this end, let $a=g(x) \in g[A \backslash E]=$ domain $\theta$. Then $\theta(a)=h(x)=g(y)$ for some $y \in A$. If $y \in A \backslash E$ we have established our claim so suppose $y \in E$. Then $h(y)=g(y)=h(x)$ so $y=x$ while $x \in A \backslash E$, a contradiction.

Since $A$ is infinite choose a sequence of disjoint subsets $\left\langle B_{m}\right\rangle_{m=0}^{\infty}$ of $A$ with $A=\bigcup_{m=0}^{\infty} B_{m}$ and each $\left|B_{m}\right|=|A|$. Let $C=h[A \backslash E] \backslash g[A \backslash E]$ and pick for each $m \in \mathbb{N}$ some function $\phi_{m}: C \stackrel{1-1}{\longrightarrow} B_{m}$. Pick $\delta: A \backslash(h[A \backslash E] \cup g[A \backslash E]) \stackrel{1-1}{\longrightarrow} B_{0}$. Pick $n$ such that $\theta^{n}=\varnothing$. Given $a \in g[A \backslash E] \cup h[A \backslash E]$, pick the first element $\mu(a) \in\{0,1, \ldots, n-1\}$ such that $\theta^{\mu(a)}(a) \notin g[A \backslash E]=\operatorname{domain}(\theta)$. (If $a \notin g[A \backslash E]$, then $\mu(a)=0$.) The assumption that $\theta^{n}=\varnothing$ says always $\mu(a)<n$.

Let $H=\left\{\left(m_{0}, m_{1}, \ldots, m_{n-1}\right) \in \mathbb{N}^{n}: m_{0}<m_{1}<\cdots<m_{n-1}\right\}$. Given $\bar{x}=\left(m_{0}, m_{1}, \ldots, m_{n-1}\right)$ in $H$, define $f_{\vec{x}} \in S$ by

$$
f_{\vec{x}}(a)= \begin{cases}\delta(a) & \text { if } a \notin h[A \backslash E] \cup g[A \backslash E] \\ \phi_{m_{i}}\left(\theta^{i}(a)\right) & \text { if } a \in g[A \backslash E] \cup h[A \backslash E] \text { and } i=\mu(a) .\end{cases}
$$

Given $a \in g[A \backslash E] \cup h[A \backslash E]$ we have $\theta^{\mu(a)}(a) \in C$ so the definition makes sense. To see that $f_{\vec{x}}$ is one-to-one one only needs to show that $\theta$ is one-to-one. To this end, let $a, b \in g[A \backslash E]$. Then $a=g(x)$ and $b=g(y)$. Assume $\theta(a)=\theta(b)$. Then $h(x)=h(y)$ so $x=y$ and $a=b$ as required. 
We now utilize Lemma 2.3. So let a finite partition $F$ of $S$ be given. Given $D \in F$, let $F(D)=\left\{\vec{x} \in H: f_{\vec{x}} \in D\right\}$. Then $\{F(D): D \in F\}$ is a finite partition of $H$ so pick by Ramsey's Theorem $\left([15]\right.$ or $\left[8\right.$, page 7]) some $D \in F$ and some $m_{0}<m_{1}<\cdots<m_{n}$ such that $\vec{x}=\left(m_{1}, m_{2}, \ldots, m_{n}\right) \in F(D)$ and $\bar{y}=\left(m_{0}, m_{1}, \ldots, m_{n-1}\right) \in F(D)$. Then $f_{\vec{x}}$ and $f_{\vec{y}}$ are in $D$. We claim that $f_{\vec{x}} \circ h=f_{\vec{y}} \circ g$ (so that $D h \cap D g \neq \varnothing$ as required).

To this end, let $a \in A$. If $a \in E$, then $h(a) \notin g[A \backslash E] \cap h[A \backslash E]$ so $f_{\vec{x}}(h(a))=\delta(h(a))=$ $\delta(g(a))=f_{\vec{y}}(g(a))$. Now assume $a \in A \backslash E$ and let $i=\mu(h(a))$. Since $h(a)=\theta(g(a))$ we have $\mu(g(a))=i \stackrel{y}{+}$. Thus $f_{\vec{x}}(h(a))=\phi_{m_{i+1}}\left(\theta^{i}(h(a))\right)=\phi_{m_{i+1}}\left(\theta^{i+1}(g(a))\right)=f_{\vec{y}}(g(a))$ as required.

Necessity. We assume essentially without loss of generality that $|A \backslash g[A \backslash E]| \leqq$ $|A \backslash h[A \backslash E]|$. (The assertion that some $\theta^{n}=\varnothing$ is equivalent to the assertion that some $\left(\theta^{-1}\right)^{n}=\varnothing$.) Pick $\delta: A \backslash g[A \backslash E] \stackrel{1-1}{\longrightarrow} A \backslash h[A \backslash E]$ and define $\tau \in S$ by

$$
\tau(a)=\left\{\begin{array}{l}
\delta(a) \text { if } a \in A \backslash g[A \backslash E] \\
\theta(a) \text { if } a \in g[A \backslash E]
\end{array}\right.
$$

Since $\theta$ is one-to-one, so is $\tau$. For each $n \in \mathbb{N}$, let $D_{n}=$ domain $\theta^{n}$. Suppose that each $D_{n} \neq \varnothing$. Define an equivalence relation $\approx$ on $S$ by $f \approx k$ if and only if there exists $n \in \mathbb{N}$ with $\left.f\right|_{D_{n}}=\left.k\right|_{D_{n}}$. Define $\phi: S / \approx \rightarrow S / \approx$ by $\phi([f])=[f \circ \tau]$. We show first that $\phi$ is well defined. Assume $f \approx k$ and pick $n \in \mathbb{N}$ such that $\left.f\right|_{D_{n}}=\left.k\right|_{D_{n}}$. We claim $\left.f \circ \tau\right|_{D_{n+1}}=$ $\left.k \circ \tau\right|_{D_{n+1}}$. To this end let $a \in D_{n+1}$ and observe that $\theta(a) \in D_{n}$. Then $f(\tau(a))=f(\theta(a))=$ $k(\theta(a))=k(\tau(a))$.

Next we show that for each $f \in S, \phi([f]) \neq[f]$. (It is here that we utilize our supposition that each $D_{n} \neq \varnothing$.) Indeed suppose we have some $n$ with $\left.f \circ \tau\right|_{D_{n}}$. Pick $a \in D_{n}$. Then $\tau(a)=\theta(a)$ so $f(a)=f(\theta(a))$. Now $a \in g[A \backslash E]$ so pick $x \in A \backslash E$ with $a=g(x)$. Then $\theta(a)=h(x)$ so $f(g(x))=f(a)=f(\theta(a))=f(h(x))$ so $g(x)=h(x)$ so $x \in E$, a contradiction.

Now by $\left[6\right.$, Lemma 9.1] pick a partition $\left\{B_{0}, B_{1}, B_{2}\right\}$ of $S / \approx$ such that for each $i$, $B_{i} \cap \phi\left[B_{i}\right]=\varnothing$. For each $i$ let $C_{i}=\cup B_{i}$ so that $\left\{C_{0}, C_{1}, C_{2}\right\}$ is a partition of $S$. By Lemma 2.3 pick $i$ such that $C_{i} g \cap C_{i} h \neq \varnothing$ and pick $f, k \in C_{i}$ with $f \circ h=k \circ g$. We show that $k \approx f \circ \tau$ so that $\phi([f])=[k]$ and hence $B_{i} \cap \phi\left[B_{i}\right]=\varnothing$, a contradiction. To see this we show $\left.k\right|_{D_{1}}=\left.f \circ \tau\right|_{D_{1}}$. Let $a \in D_{1}$ and pick $x \in A \backslash E$ such that $a=g(x)$. Then $f(\tau(a))=$ $f(\theta(a))=f(\theta(g(x)))=f(h(x))=k(g(x))=k(a)$.

We see now that half of the characterization of Theorem 2.9 is valid in any left cancellative semigroup.

Corollary 2.10. Let $(S,)^{-}$be a left cancellative semigroup, let $x, y \in S$ and let $E=$ $\{a \in S: x a=y a\}$. Define $\theta: x \cdot(S \backslash E) \rightarrow y \cdot(S \backslash E)$ by $\theta(x \cdot a)=y \cdot a$ for $a \in S \backslash E$. If there exists $p$ in $\beta S$ such that $p \cdot x=p \cdot y$, then for some $n \in \mathbb{N}, \theta^{n}=\varnothing$.

Proof. If $E=S$, then $\theta=\varnothing$ so we can presume $E \neq S$. Let $M=\{f: f: S \stackrel{1-1}{\longrightarrow} S\}$. Define $\phi: S \rightarrow M$ by $\phi(z)=\lambda_{z}$ for all $z \in S$. Then $\phi$ is a homomorphism of $S$ to $M$ so $\phi$ extends to a homomorphism $\phi^{\beta}: \beta S \rightarrow \beta M$. Observe that $E=\left\{a \in S: \lambda_{x}(a)=\lambda_{y}(a)\right\}$. Observe further 
that $\theta: \lambda_{x}[S \backslash E] \rightarrow \lambda_{y}[S \backslash E]$ and is defined so that $\theta\left(\lambda_{x}(a)\right)=\lambda_{y}(a)$ for $a \in S \backslash E$. Since $\phi^{\beta}(p) \lambda_{x}=\phi^{\beta}(p \cdot x)=\phi^{\beta}(p \cdot y)=\phi^{\beta}(p) \lambda_{y}$ we have by Theorem 2.9 that for some $n \in \mathbb{N}$, $\theta^{n}=\varnothing$.

Corollary 2.11. Let $(S, \cdot)$ be a left cancellative semigroup and let $x, y \in S$ such that $x \cdot y=y \cdot x$ but $x \neq y$. Then for all $p \in \beta S, p \cdot x \neq p \cdot y$.

Proof. Let $E$ and $\theta$ be as in Corollary 2.10. Note that for each $n$ and $k$ in $\mathbb{N}$, $y^{k} x^{n} \notin E$. Indeed if $x y^{k} x^{n}=y y^{k} x^{n}$, then $y^{k} x^{n} x=y^{k} x^{n} y$ so that $x=y$. Further $\theta\left(y^{k} x^{n}\right)=$ $y^{k+1} x^{n-1}$. Consequently, for each $n \in \mathbb{N}, x^{n+1} \in \operatorname{domain}\left(\theta^{n}\right)$.

Corollary 2.12. Let $(S, \cdot)$ be a left cancellative semigroup and let $x, y \in S$. Assume there is some $B \subseteq S$ such that $y B \subseteq x B$ and there is some $s \in B$ with $y \cdot s \neq x \cdot s$. Then for all $p \in \beta S$, $p \cdot x \neq p \cdot y$.

Proof. Let $E$ and $\theta$ be as in Corollary 2.10. Let $\gamma=\left.\theta\right|_{x \cdot(B \backslash E)}$. We show that range $\gamma \subseteq$ domain $\gamma$ so that for all $n \in \mathbb{N}, \gamma^{n} \neq \varnothing$ (and hence $\theta^{n} \neq \varnothing$ ). Indeed let $a \in$ range $\gamma$ and pick $b \in(B \backslash E)$ such that $a=\gamma(x \cdot b)=y \cdot b$. Then $a=y \cdot b \in y \cdot B \subseteq x \cdot B$. Suppose $a \in x \cdot E$. Then $y \cdot b=x \cdot c$ for some $c \in E$ so $y \cdot b=x \cdot c=y \cdot c$ so $b=c$, a contradiction. Thus $a \in X \cdot(B \backslash E)$ as required.

Theorem 2.9 provides us with plentiful examples of left cancellative semigroups $S$ and points $x \neq y$ in $S$ such that $p \cdot x=p \cdot y$ for some $p \in \beta S$. These examples are all large however, having at least $2^{\omega}$ elements. Especially in view of the special role of countable semigroups in Theorem 2.2 , it is reasonable to ask whether one can find such examples with $S$ countable.

Theorem 2.13. There exist a countable left cancellative semigroup $S$ and distinct elements $g$ and $h$ in $S$ and $p \in \beta S$ such that $p g=p h$.

Proof. Let $S=\{f: f: \mathbb{N} \rightarrow \mathbb{N}$ and $f$ is one-to-one and there exist $m$ and $r$ in $\mathbb{N}$ such that $\left.\forall n \geqq m, f(n)=2^{r} \cdot n\right\}$. Observe that $S$ is indeed countable and is a semigroup under composition. Define $g$ and $h$ in $S$ as follows: If $n \geqq 2, g(n)=h(n)=2 n$, while $g(1)=1$ and $h(1)=2$.

Now let $F$ be a finite partition of $S$. By Lemma 2.3 it suffices to show that some $A \in F$ has $A g \cap A h \neq \varnothing$. Let $E=\{f \in S: f(1)$ and $f(2)$ are odd and $f(1)<f(2)$ and for all $n \geqq 3$, $f(n)=2 n\}$. For $A \in F$, let $\hat{A}=\{(f(1), f(2)): f \in E \cap A\}$. Given odd integers $a<b$ there is a unique member of $E$ with $(f(1), f(2))=(a, b)$ so $\{(a, b): a$ and $b$ are odd and $a<b\}=$ $\bigcup_{A \in F} \hat{A}$. Pick by Ramsey's Theorem [15] some $A \in F$ and some odd integers $a, b, c$ with $a<b<c$ and $\{(a, b),(a, c),(b, c)\} \subseteq \hat{A}$. Pick $k$ and $f$ in $E \cap A$ with $(a, b)=(k(1), k(2))$ and $(b, c)=(f(1), f(2))$. Then $f(g(1))=f(1)=b=k(2)=k(h(1)) \quad$ and $\quad$ for $n \geqq 2$, $f(g(n))=f(2 n)=4 n=k(2 n)=k(h(n))$. Thus $A g \cap A h \neq \varnothing$.

\section{The condition $p \notin p \cdot S^{*}$}

One of the most useful characterizations of the left cancellability of $p$ in $(\beta \mathbb{N},+)$ is the 
requirement that $p \notin p+\mathbb{N}^{*}$. We investigate in this section conditions under which $p \notin p \cdot S^{*}$ (or " $p \notin p \cdot S^{*}$ and $\lambda_{p}$ is one-to-one on $S^{\prime \prime}$ ) can be added to the characterizations of left cancellability of $p$ in $\beta S$. The reason for working with $S^{*}$ rather than with $\beta S$ is that if $a$ is any right identity of $S$, then for all $p \in \beta S, p=p \cdot a$.

Theorem 3.1. Let $(S, \cdot)$ be a semigroup and for each $x \in S$ let $F_{x}=\{y \in S: y \cdot x=x\}$. If there is some finite $G \subseteq S$ such that $\bigcap_{x \in G} F_{x}$ is finite and if $p \in \beta S$ is such that $\lambda_{p}$ is one-to-one on $\beta S$, then $p \notin p \cdot S^{*}$.

Proof. Suppose one has some $q \in S^{*}$ such that $p=p \cdot q$. Since $\bigcap_{x \in G} F_{x}$ is finite, pick some $x \in G$ such that $F_{x} \notin q$. Then $x \neq q \cdot x$ while $p \cdot(q \cdot x)$, is a contradiction.

We observe that even in a left cancellative semigroup, $\lambda_{p}$ being one-to-one on $\beta S$ need not imply $p \notin p \cdot S^{*}$. Indeed let $S$ be any infinite right zero semigroup (wherein $x \cdot y=y$ for all $x$ and $y$ ). Then $\beta S$ is also right zero so for any $p \in S^{*}, \lambda_{p}$ is one-to-one while $p=p \cdot p \in p \cdot S^{*}$.

Theorem 3.2. Let $(S, \cdot)$ be a right cancellative semigroup, let $p \in S^{*}$. Assume that for all $q \in S^{*}$ and all $a \in S$, if $p \cdot a=p \cdot q$, then for all $A \in q, A / a$ is infinite. If $p \notin p \cdot S^{*}$ and $\lambda_{p}$ is one-to-one on $S$, then $\{p \cdot x: x \in S\}$ is discrete (and $\lambda_{p}$ is one-to-one on $S$ ).

Proof. Let $a \in S$ and suppose $p \cdot a \in c l\{p \cdot x: x \in S \backslash\{a\}\}=p \cdot c l\{x: x \in S \backslash\{a\}\}$. Pick $q \in$ $c l\{x: x \in S \backslash\{a\}\}$ such that $p \cdot a=p \cdot q$. Since $\lambda_{p}$ is one-to-one on $S, q \notin S$ so $q \in S^{*}$. Consider $\mathscr{A}=\{A / a: A \in q\}$. By our assumption (and the fact that $(A \cap B) / a=(A / a) \cap(B / a))$ one has that any finite subfamily of $\mathscr{A}$ has infinite intersection. Consequently we may pick some $r \in S^{*}$ with $\mathscr{A} \subseteq r$.

We claim $p=p \cdot r$. To this end let $B \in p$. Then $B \cdot a \in p \cdot a=p \cdot q$ so $A \in q$ where $A=\{x \in S:(B \cdot a) / x \in p\}$. Then $A / a \in r$. We show that $A / a \subseteq\{x \in S: B / x \in p\}$ and hence that $B \in p \cdot r$ as required. Let $x \in A / a$. Then $x \cdot a \in A$ so $(B \cdot a) /(x \cdot a) \in p$. Since $S$ is right cancellative $(B \cdot a) /(x \cdot a) \subseteq B / x$ so that $B / x \in p$ as required.

Corollary 3.3. Let $(S, \cdot)$ be a countable group and let $p \in S^{*}$. Then $\lambda_{p}$ is one-to-one on $\beta S$ if and only if $p \notin p \cdot S^{*}$.

Proof. Necessity. For each $x \in S,\{y \in S: y \cdot x=x\}$ has only one element so Theorem 3.1 applies.

Sufficiency. Given $q \in S^{*}$ and $A \in q, A$ is infinite so for all $a \in S, A / a$ is infinite. By Theorem $2.5 \lambda_{p}$ is one-to-one on $S$ so by Theorem 3.2, $\{p+x: x \in S\}$ is discrete. Thus Theorem 2.2 applies.

Corollary 3.4. Consider the semigroup $(\mathbb{N}, \cdot)$. Let $p \in \mathbb{N}^{*}$. Then $\lambda_{p}$ is one-to-one on $\beta \mathbb{N}$ if and only if $p \notin p \cdot \mathbb{N}^{*}$.

Proof. Necessity. Given $x \in \mathbb{N},\{y \in \mathbb{N}: y \cdot x=x\}=\{1\}$ so Theorem 3.1 applies. 
Sufficiency. By Theorem $2.5 \lambda_{p}$ is one-to-one on $\mathbb{N}$ so it suffices to show that the assumption of Theorem 3.2 is valid. (Then Theorem 2.2 applies.) To this end let $q \in \mathbb{N}^{*}$, let $a \in \mathbb{N}$, assume $p \cdot a=p \cdot q$, and let $A \in q$. Suppose that $A / a$ is finite. Then $\mathbb{N} a \notin q$. (For otherwise one has that $A \cap \mathbb{N a}$ is infinite and consequently $A / a$ is infinite.) In particular $a \neq 1$. Write $a=\prod_{i=1}^{m} r_{i}^{\alpha_{i}}$ where each $r_{i}$ is prime and $r_{i} \neq r_{j}$ for $i \neq j$. Now $\mathbb{N} a=\bigcap_{i=1}^{m} \mathbb{N} r_{i}^{\alpha_{i}}$ and consequently we may pick $i$ such that $\mathbb{N} r_{i}^{\alpha_{i}} \notin q$. Define $\phi: \mathbb{N} \rightarrow \omega$ by $\phi(x)=j$ if and only if $r_{i}^{j}$ divides $x$ and $r_{i}^{j+1}$ does not divide $x$. Pick $j \in\left\{0,1, \ldots, \alpha_{i}\right\}$ such that $B=\left\{x: \phi(x) \equiv j \bmod \left(\alpha_{i}+1\right)\right\} \in p$. Then $B \cdot a \in p \cdot a=p \cdot q$ so $\{x \in \mathbb{N}:(B \cdot a) / x \in p\} \in q$. Pick $x \in \mathbb{N} \backslash \mathbb{N} r_{i}^{\alpha_{i}}$ such that $(B \cdot a) / x \in p$. Pick $y \in B \cap(B \cdot a) / x$. Now $y \in B$ so $\phi(y) \equiv j \bmod \left(\alpha_{i}+1\right)$ while $y \cdot x \in B \cdot a$ so $\phi(y)+\phi(x)=\phi(y \cdot x) \equiv j+\alpha_{i} \bmod \left(\alpha_{i}+1\right)$. Thus $\phi(x) \equiv \alpha_{i} \bmod \left(\alpha_{i}+1\right)$ while $\phi(x) \in\left\{0,1, \ldots, \alpha_{i}-1\right\}$, a contradiction.

We utilize Corollary 3.3 to produce a simple characterization of left cancellability in $(\beta \mathbb{Z},+)$. Here we pretend that $\beta \mathbb{N} \subseteq \beta \mathbb{Z}$ and brush over the distinction between an ultrafilter on $\mathbb{N}$ and an ultrafilter on $\mathbb{Z}$ with $\mathbb{N}$ as a member. Given a point $p$ in $c l_{\beta \mathrm{Z}}(-\mathbb{N})$ one has left cancellation holds at $p$ in $\beta \mathbb{Z}$ if and only if it holds at $-p=\{-A: A \in p\}$. Consequently Corollary 3.6 provides a complete characterization of left cancellability in $\beta \mathbb{Z}$.

Lemma 3.5. Let $S$ be a semigroup and let $p \in S^{*}$. Let $E \subset S$. Then $p \notin p \cdot c l E$ if and only if there is some $A \in p$ such that $A / a \notin p$ for all $a \in E$.

Proof. $p \notin p \cdot c l E$

$\Leftrightarrow p \notin \operatorname{cl}(p \cdot E)$

$\Leftrightarrow$ there exists $A \in p$ such that $\bar{A} \cap(p \cdot E)=\varnothing$

$\Leftrightarrow$ there exists $A \in p$ such that $A / a \notin p$ for all $a \in E$.

Corollary 3.6. Let $p \in \mathbb{N}^{*}$. Then $\lambda_{p}$ is one-to-one on $\beta \mathbb{Z}$ if and only if there is an increasing sequence $\left\langle x_{n}\right\rangle_{n=1}^{\infty}$ in $\mathbb{N}$ such that for each $k \in \mathbb{N},\left\{x_{n}: x_{n+1}\right\rangle x_{n}+k$ and $\left.x_{n}>x_{n-1}+k\right\} \in p$.

Proof. Note that $\{a \in \mathbb{Z}: p \neq p+a\}=\mathbb{Z} \backslash\{0\}$. Since $p \notin p+(\mathbb{Z} \backslash\{0\})$ (an easy fact, or see [11, Lemma 2.4]) we have by Lemma 3.5 that $p \notin p+\mathbb{Z}^{*}$ if and only if there is some $A \in p$ such that $A-a \notin p$ for all $a \in \mathbb{Z} \backslash\{0\}$. Consequently by Corollary 3.3 , it suffices to show that there is an increasing sequence $\left\langle x_{n}\right\rangle_{n=1}^{\infty}$ in $\mathbb{N}$ such that for each $k \in \mathbb{N}$, $\left\{x_{n}: x_{n+1}>x_{n}+k\right.$ and $\left.x_{n}>x_{n-1}+k\right\} \in p$ if and only if there exists $A$ in $p$ such that $A-a \notin p$ for all $a \in \mathbb{Z} \backslash\{0\}$.

Sufficiency. We may assume $A \subseteq \mathbb{N}$. Enumerate $A$ in increasing order as $\left\{x_{n}: n \in \mathbb{N}\right\}$. Suppose that for some $k \in \mathbb{N} \quad\left\{x_{n}: x_{n+1} \leqq x_{n}+k\right.$ or $\left.x_{n} \leqq x_{n-1}+k\right\} \in p$. Then $\bigcup_{a=1}^{k}\left(\left\{x_{n}: x_{n+1}=x_{n}+a\right\} \cup\left\{x_{n}: x_{n}=x_{n-1}+a\right\} \in p\right)$. Pick $a$ such that $\left\{x_{n}: x_{n+1}=x_{n}+a\right\} \in p$ or $\left\{x_{n}: x_{n}=x_{n-1}+a\right\} \in p$. In the first case $A-a \in p$. In the second case $A-(-a) \in p$. In either case we have a contradiction. 
Necessity. Let $A=\left\{x_{n}: n \in \mathbb{N}\right\}$. If for some $a \in \mathbb{Z} \backslash\{0\}$ one has $A-a \in p$ then $\left\{x_{n}: x_{n+1}=\right.$ $\left.x_{n}+a\right\} \in p($ if $a>0)$ or $\left\{x_{n}: x_{n}=x_{n-1}+(-a)\right\} \in p$ (if $\left.a<0\right)$.

Condition (h) of Theorem 2.1 and the condition of Corollary 3.6 certainly appear to be different. It is not however obvious that they are not equivalent. We see now that they are not.

Theorem 3.7. There is a point $p \in \mathbb{N}^{*}$ such that $\lambda_{p}$ is one-to-one on $\beta \mathbb{N}$ but $\lambda_{p}$ is not one-to-one on $\beta \mathbb{Z}$.

Proof. Let $M=F S\left(\left\langle 2^{2 n}\right\rangle_{n=1}^{\infty}\right)$ and pick an indempotent $q \in \bar{M}$. (The set $\bigcap_{m=1}^{\infty} \overline{F S\left(\left\langle 2^{2 n}\right\rangle_{n=m}^{\infty}\right)}$ is a compact subsemigroup which therefore has idempotents by [7, Corollary 2.10].) Let $p=q+(-q)$ where $-q=\{-A: A \in q\}$. Then $-q$ is an idempotent of $\beta \mathbb{Z}$ so $p+(-q)=q+(-q)+(-q)=q+(-q)=p$ so $p \in p+\mathbb{Z}^{*}$ so by Corollary $3.3 \lambda_{p}$ is not one-to-one on $\beta \mathbb{Z}$.

Now let $A=\left\{\sum_{n \in F} 2^{2 n}-\sum_{n \in G} 2^{2 n}: F\right.$ and $G$ are finite nonempty subsets of $\omega$ and $\max G<\min F\}$. Then $A \in p$. Notice further that for $x \in \mathbb{N}, x \in A$ if and only if there exist even integers $a<b$ such that the binary expansion of $x$ has (1) only 0's to the right of $a$ (and not at $a$ ), (2) 0's only in even positions between $a$ and $b$, and (3) 1's only in even positions to the left of $b$ (but not at $b$ ). That is, if $x=\sum_{n \in H} 2^{n}$ one has $\min H=a, b \notin H$, $\{a, a+1, \ldots, b\} \backslash H \subseteq \mathbb{N} 2$, and $H \backslash\{1,2, \ldots, b\} \subseteq \mathbb{N} 2$. We now claim that for all $k \in \mathbb{N}$, $A-k \notin p$ so that by Lemma $3.5, p \notin p+\beta \mathbb{N}$ and hence by Theorem $2.1 \lambda_{p}$ is one-to-one on $\beta \mathbb{N}$. To see this suppose instead we have some $k=\sum_{n \in H} 2^{n}$ with $A-k \in p=q+(-q)$. Now $B=\left\{\sum_{n \in G}-2^{2 n}: G\right.$ is a finite nonempty subset of $\mathbb{N}$ and $\left.\min G>\max H+1\right\} \in-q$ so pick $y=\sum_{n \in G}-2^{2 n}$ in $B \cap\{y:(A-k)-y \in q\}$. Then $C=\left\{\sum_{n \in F} 2^{2 n}: F\right.$ is a finite nonempty subset of $\mathbb{N}$ and $\min F>\max G\} \in q$ so pick $z=\sum_{n \in F} 2^{2 n}$ in $C \cap((A-k)-y)$. Then $z+y+k=\sum_{n \in F} 2^{2 n}-\sum_{n \in G} 2^{2 n}+\sum_{n \in H} 2^{n}$. A brief consideration of the resulting binary expansions shows that $z+y+k \notin A$, a contradiction.

The hypothesis of Theorem 3.2 seems a bit strong. We see now that some reasonably strong assumption is needed.

Theorem 3.8. There is a countable cancellative commutative semigroup $(S,+)$ such that $\lambda_{p}$ is one-to-one on $S$ for all $p \in \beta S$ and there exists $p \in S^{*}$ such that $p \notin p+S^{*}$ but $\lambda_{p}$ is not one-to-one on $\beta S$.

Proof. Let $S=\mathbb{N} \times \mathbb{N}$ with ordinary addition. By Theorem 2.4 we have $\lambda_{p}$ is one-to-one on $S$ for all $p \in \beta S$.

We now show that there is a homomorphism $f: \beta \mathbb{N} \times \mathbb{N} \rightarrow \beta S$ such that $f$ is the identity on $\mathbb{N} \times \mathbb{N}$ and $f\left[\mathbb{N}^{*} \times \mathbb{N}\right] \subseteq S^{*}$. Given $n \in \mathbb{N}$, consider $g_{n}(m)=(m, n)$. Then $g_{n}$ has a continuous extension $g_{n}^{\beta}: \beta \mathbb{N} \rightarrow \beta S$ and if $p \in \mathbb{N}^{*}, g_{n}^{\beta}(p) \in S^{*}$. For $p \in \beta \mathbb{N}$ and $n \in \mathbb{N}$ we define $f(p, n)=g_{n}^{\beta}(p)$. Observe that given $q \in \beta \mathbb{N}, n \in \mathbb{N}$, and $A \subseteq S$ one has that $A \in f(q, n)$ if and only if there exists $B \in q$ with $B \times\{n\} \subseteq A$. Using this observation one easily establishes that $f$ is a homomorphism and $f\left[\mathbb{N}^{*} \times \mathbb{N}\right] \subseteq S^{*}$.

Now pick some $p \in \mathbb{N}^{*}$ with $p \in p+\mathbb{N}^{*}$. For example, we may pick by $[7$, 
Corollary 2.10] some $p \in \mathbb{N}^{*}$ with $p=p+p$. Then $f(p, 1)+(1,1)=$ $f(p, 1)+f(1,1)=f(p+1,2)=f(p+p+1,2)=f(p, 1)+f(p+1,1)$. Since $f(p+1,1) \in S^{*}$ we have $\lambda_{f(p .1)}$ is not one-to-one on $\beta S$.

To conclude the proof we show that $f(p, 1) \notin \beta S+\beta S$, so in particular $f(p, 1) \notin f(p, 1)+S^{*}$. To this end, suppose that $f(p, 1)=q+r$ for some $q$ and $r$ in $\beta S$. Now $\mathbb{N} \times\{1\} \in f(p, 1)$ so $\{(x, y):(\mathbb{N} \times\{1\})-(x, y) \in q\} \in r \quad$ so pick $(x, y)$ with $(\mathbb{N} \times\{1\})-(x, y) \in q$. Pick $(w, z) \in(\mathbb{N} \times\{1\})-(x, y)$. Then $z, y \in \mathbb{N}$ while $z+y=1$, a contradiction.

\section{Right cancellation in $(\beta \mathbb{N},+)$}

We specialize now to the semigroup $(\beta \mathbb{N},+)$, obtaining several sufficient conditions for right cancellation to hold at a point $p$ of $(\beta \mathbb{N},+)$.

Theorem 4.1. Let $p \in \mathbb{N}^{*}$. The following statements are equivalent:

(a) $\rho_{p}$ is one-to-one on $\beta \mathbb{N}$;

(b) $\rho_{p}$ is one-to-one on $\bigcap_{n=1}^{\infty} \mathrm{cl}(\mathbb{N} n)$;

(c) There exist a first countable topological group $(G,+)$ and a continuous homomorphism $h: \beta \mathbb{N} \rightarrow G$ such that $\rho_{p}$ is one-to-one on $\operatorname{ker}(h)$.

Proof. That (a) implies (b) is trivial. To see that (b) implies (c), let $G=\bigotimes_{n=1}^{\infty} \mathbb{Z}_{n}$ and define $h: \mathbb{N} \rightarrow G$ so that for all $m$ and $n$ in $\mathbb{N}, h(m)(n) \equiv m(\bmod n)$. Denote by $h^{\beta}$ the continuous extension to $\beta \mathbb{N}$, where $G$ is given the product topology and each $\mathbb{Z}_{n}$ is discrete. The $G$ is a first countable topological group. It is well known that as the continuous extension of a homomorphism, $h^{\beta}$ is a homomorphism on $\beta \mathbb{N}[14$, Corollary 2.3].

We now show that (c) implies (a) so let such $G$ and $h$ be given. Let $q, r \in \beta \mathbb{N}$ and assume that $q+p=r+p$. Then $h(q+p)=h(r+p)$ so $h(q)+h(p)=h(r)+h(p)$ so $h(q)=h(r)$. Let $\alpha=h(r)$. We claim that we can find $s \in \mathbb{N}^{*}$ such that $\lambda_{s}$ is one-to-one an $\beta \mathbb{N}$ and $h(2)=-\alpha$. This will suffice for $s+q$ and $s+r$ are in $\operatorname{ker}(h)$ and $s+q+p=s+r+p$ so that $s+q=s+r$ and hence $q=r$.

It is well known from compact semigroup theory that $h[\beta \mathbb{N}]$ is a group since it is the compact closure of a commutative subsemigroup of $G$. (An argument is presented in the introduction to [1]. An even simpler argument establishes that any compact cancellative semitopological semigroup $S$ is a group: Any idempotent will be an identity since $e e=e$ implies that for any $x e e x=e x$ and hence $e x=x$. Thus there is a unique idempotent which must be minimal [3]. Therefore $S=e S e$ is a group.) Then $h\left[\mathbb{N}^{*}\right]$ is a compact subsemigroup of $h[\beta \mathbb{N}]$ which therefore contains $c l\{\alpha, \alpha+\alpha, \alpha+\alpha+\alpha, \ldots\}$. This latter set is a homomorphic image of $\beta \mathbb{N}$ which we again conclude is a group and must therefore contain $-\alpha$. Pick $t \in \mathbb{N}^{*}$ such that $h(t)=-\alpha$. Let $\left\langle U_{n}\right\rangle_{n=1}^{\infty}$ be a neighbourhood basis for $-\alpha$ in $G$ with $U_{n+1} \subseteq U_{n}$ for each $n$. For each $n$ let $A_{n}=\left\{x \in \mathbb{N}: h(x) \in U_{n}\right\}$. Then each 
$A_{n} \in t$ so each $A_{n}$ is infinite. Pick $x_{n} \in A_{n}$ for each $n$ with $x_{n+1}>x_{n}+n$. Pick $s \in \mathbb{N}^{*}$ with $\left\{x_{n}: n \in \mathbb{N}\right\} \in s$. By Theorem $2.1 \lambda_{s}$ is one-to-one on $\beta S$. Since $h(s)=-\alpha$, we are done.

Note that in the following theorem we make no assumptions about a relationship between the operation on $S$ and its topology, beyond the ability to support a continuous homomorphism from $\beta \mathbb{N}$.

Theorem 4.2. Let $(S,+)$ be a cancellative semigroup with a topology and let $h: \beta \mathbb{N} \rightarrow S$ be a continuous function such that for all $q \in \beta \mathbb{N}$ and all $r \in \bigcap_{n=1}^{\infty} \mathbb{N} n, h(r+q)=h(r)+h(q)$. Let $\left\langle b_{n}\right\rangle_{n=1}^{\infty}$ be a sequence in $\beta \mathbb{N}$ such that $\rho_{b_{n}}$ is one-to-one for each $n \in \mathbb{N}, h\left(b_{n}\right) \neq h\left(b_{m}\right)$ for $n \neq m$, and $\left\{h\left(b_{n}\right): n \in \mathbb{N}\right\}$ is discrete. If $p$ is a limit point of $\left\{b_{n}: n \in \mathbb{N}\right\}$, then $\rho_{p}$ is one-to-one.

Proof. By Theorem 4.1 it suffices to let $q, r \in \bigcap_{n=1}^{\infty} \overline{\mathbb{N} n}$ with $q+p=r+p$ and show that $q=r$. Then $\left(q+c l\left\{b_{n}: n \in \mathbb{N}\right\}\right) \cap\left(r+c l\left\{b_{n}: n \in \mathbb{N}\right\}\right) \neq \varnothing$ so by Lemma 1.1 we may without loss of generality pick $n \in \mathbb{N}$ and $t \in c l\left\{b_{n}: n \in \mathbb{N}\right\}$ such that $q+b_{n}=r+t$. Now $h(q)+h(p)=h(q+p)=h(r+p)=h(r+p)=h(r)+h(p)$ so by right cancellation in $S$, $h(q)=h(r)$. Since also $h(q)+h\left(b_{n}\right)=h(r)+h(t)$ we have by left cancellation in $S$ that $h\left(b_{n}\right)=h(t)$. Since $\left\{h\left(b_{m}\right): m \in \mathbb{N}\right\}$ is discrete, pick a neighbourhood $V$ of $h\left(b_{n}\right)$ which misses $\left\{h\left(b_{m}\right): m \in \mathbb{N} \backslash\{n\}\right\}$ and pick a member $B$ of $t$ such that $h[B] \subseteq V$. Given any $m \in \mathbb{N}$ such that $b_{m} \in B$, one has $h\left(b_{m}\right) \in V$ so $m=n$. That is we must have $t=b_{n}$. Then $q+b_{n}=r+b_{n}$ so by right cancellation at $b_{n}, q=r$.

We remark that functions as in the hypothesis of Theorem 4.2 are easy to come by. Indeed let $S$ be the non-negative reals and let $\left\langle c_{n}\right\rangle_{n=1}^{\infty}$ be any sequence of positive reals such that $\sum_{n=1}^{\infty} c_{n}$ converges. Given $x=\sum_{n \in F} 2^{n}$, define $h(x)=\sum_{n \in F} c_{n}$. Since $h$ is bounded by $\sum_{n=1}^{\infty} c_{n}$, it has a continuous extension to $\beta \mathbb{N}$ which is easily seen to satisfy the hypothesis.

Some applications of Theorem 4.2 yield easily described sufficient conditions for right cancellation.

Corollary 4.3. Let $\left\langle b_{n}\right\rangle_{n=1}^{\infty}$ be a one-to-one sequence in $\mathbb{N}$ and assume there is a sequence $\left\langle a_{n}\right\rangle_{n=1}^{\infty}$ such that for each $n \in \mathbb{N},\left\{m \in \mathbb{N}: a_{n}\right.$ divides $\left.b_{m}-b_{n}\right\}$ is finite. If $p \in c l\left\{b_{n}: n \in \mathbb{N}\right\}$ then $\rho_{p}$ is one-to-one.

Proof. If $p=b_{n}$ for some $n$, the conclusion is immediate so we assume $p \in \mathbb{N}^{*}$. Let $G=\bigotimes_{n=1}^{\infty} \mathbb{Z}_{n}$ and let $h: \beta \mathbb{N} \rightarrow G$ be the natural homomorphism (as described in the proof of Theorem 4.1). One easily sees that $h$ and $\left\langle b_{n}\right\rangle_{n=1}^{\infty}$ satisfy all hypotheses of Theorem 4.2 except possibly the requirement that $\left\{h\left(b_{n}\right): n \in \mathbb{N}\right\}$ is discrete. (Since each $b_{n} \in \mathbb{N}$ one has each $\rho_{b_{n}}$ is one-to-one.) To see this let $n \in \mathbb{N}$ and let $V=\left\{u \in G: u\left(a_{n}\right)=h\left(b_{n}\right)\left(a_{n}\right)\right\}$. Then $V$ is a basic neighbourhood of $h\left(b_{n}\right)$ in $G$. If $h\left(b_{m}\right) \in V$ then $a_{n}$ divides $b_{m}-b_{n}$ so $V$ contains only finitely many members of $\left\{h\left(b_{m}\right): m \in \mathbb{N}\right\}$.

Corollary 4.4. Let $\left\langle b_{n}\right\rangle_{n=1}^{\infty}$ be a one-to-one sequence in $\mathbb{N}$. If either

(a) for all $n<m$ in $\mathbb{N}, b_{n}$ divides $b_{m}$; or 
(b) for all $n<m$ in $\mathbb{N}, b_{n}$ does not divide $b_{m}$

then for each $p \in c l\left\{b_{n}: n \in \mathbb{N}\right\}, \rho_{p}$ is one-to-one.

Proof. If hypothesis (a) holds let $a_{n}=b_{n+1}$ for each $n$. If hypothesis (b) holds let $a_{n}=b_{n}$ for each $n$. In either case the hypotheses of Corollary 4.3 are satisfied.

Corollary 4.5. The set $D=\left\{p \in \mathbb{N}^{*}: \lambda_{p}\right.$ and $\rho_{p}$ are one-to-one on $\left.\beta \mathbb{N}\right\}$ has dense interior in $\mathbb{N}^{*}$.

Proof. Let $q \in \mathbb{N}^{*}$ and let $A \in q$. We show that $\bar{A} \cap$ int $_{N^{*}} D \neq \varnothing$. Pick a sequence $\left\langle x_{n}\right\rangle_{n=1}^{\infty}$ in $A$ such that for each $\left.n, x_{n+1}\right\rangle x_{n}+n$. By Theorem $2.1(h)$ if $\left\{x_{n}: n \in \mathbb{N}\right\} \in p$, then $\lambda_{p}$ is one-to-one on $\beta \mathbb{N}$. Now let $B=\left\{\left\{x_{n}, x_{m}\right\}: n<m\right.$ and $x_{n}$ divides $\left.x_{m}\right\}$ and let $C=\left\{\left\{x_{n}, x_{m}\right\}: n<m\right.$ and $x_{n}$ does not divide $\left.x_{m}\right\}$. Pick by Ramsey's Theorem [15] an increasing subsequence $\left\langle b_{n}\right\rangle_{n=1}^{\infty}$ of $\left\langle x_{n}\right\rangle_{n=1}^{\infty}$ such that either (a) $\left\{\left\{b_{n}, b_{m}\right\}: n<m\right\} \subseteq B$ or (b) $\left\{\left\{b_{n}, b_{m}\right\}: n<m\right\} \subseteq C$. In either case, by Corollary 4.4. we have that $\rho_{p}$ is one-to-one whenever $p \in\left(c l\left\{b_{n}: b \in \mathbb{N}\right\}\right) \cap \mathbb{N}$.

Of course, if $p$ is in the smallest ideal $K(\beta \mathbb{N},+)$ of $(\beta \mathbb{N},+)$ then both $\lambda_{p}$ and $\rho_{p}$ fail to be one-to-one. (For then $R=p+\beta \mathbb{N}$ and $L=\beta \mathbb{N}+p$ are minimal right and left ideals respectively [3, Theorem 1.3.11]. Then $R=p+R$ and $L=L+p$ so for any $q \in \beta \mathbb{N} \backslash K(\beta \mathbb{N})$ then there exist $r \in R$ and $l \in L$ such that $p+q=p+r$ and $q+p=l+p$.) The closure of $K(\beta \mathbb{N},+)$ consists of all ultrafilters $p$ for which each $A \in p$ is piecewise syndetic [10, Theorem 3.8]. (The set $A \subseteq \mathbb{N}$ is piecewise syndetic if and only if there is some $k \in \mathbb{N}$ with $d^{*}\left(\bigcup_{t=1}^{k} A-t\right)=1$ where $d^{*}(B)=\sup \left\{\alpha \in \mathbb{R}\right.$ : there are increasing sequences $\left\langle z_{n}\right\rangle_{n=1}^{\infty}$ and $\left\langle t_{n}\right\rangle_{n=1}^{\infty}$ such that for all $\left.n \in \mathbb{N},\left|B \cap\left\{z_{n}+1, z_{n}+2, \ldots, z_{n}+t_{n}\right\}\right| \geqq \alpha t_{n}\right\}$.) It is known [10, Theorem 4.6] that there exist points $p$ in $\operatorname{cl} K(\beta \mathbb{N},+)$ for which $\lambda_{p}$ is one-to-one. The next result shows that we can get at least reasonably close to $K(\beta \mathbb{N},+)$ with points at which $\rho_{p}$ is one-to-one.

Theorem 4.6. Let $\varepsilon>0$ be given. There exists $X \subseteq \mathbb{N}$ with $d^{*}(X)>1-\varepsilon$ such that $\rho_{p}$ is one-to-one for each $p \in \bar{X}$,

Proof. Pick an increasing sequence $\langle l(n)\rangle_{n=1}^{\infty}$ such that $\sum_{n=1}^{\infty}(1 /(l(n)) !)<\varepsilon$. Let $X_{1}=\mathbb{N}$ and let $b_{1}=\min X_{1}$. Inductively given $X_{n}$, let $b_{n}=\min \left(X_{n} \backslash\left\{b_{1}, b_{2}, \ldots, b_{n-1}\right\}\right)$. Let $k(n)=\left(\max \left\{l(n), b_{n}, k(n-1)+1\right\} \quad\right.$ and let $\quad X_{n+1}=X_{n} \backslash\left(\mathbb{N} \cdot k(n) !+b_{n}\right)$. Let $X=\bigcap_{n=1}^{\infty} X_{n}=\left\{b_{n}: n \in \mathbb{N}\right\}$. Observe that if $m<n$ then $\left(\mathbb{N} \cdot k(n) !+b_{n}\right) \cap\left(\mathbb{N} \cdot k(m) !+b_{m}\right)=\varnothing$. (For if $a \cdot k(n) !+b_{n}=b \cdot k(m) !+b_{m}$, then $b_{n}=\left(b-((a \cdot k(n) !) / k(m) !) \cdot k(m) !+b_{m}\right.$ so $\left.b_{n} \notin X_{m+1} \supseteq X_{n}\right)$ Consequently, given $n$ and $a \leqq((k(n+1) !) / k(n))$, we have that

so that

$$
|X \cap\{1,2, \ldots, a \cdot k(n) !\}|=a \cdot k(n) !-\sum_{m=1}^{n}\left(\frac{a \cdot k(n) !}{k(m) !}-1\right)
$$




$$
|X \cap\{1,2, \ldots, a \cdot k(n) !\}| /(a \cdot k(n) !)=1+n /(a \cdot k(n)) !-\sum_{m=1}^{n} \frac{1}{k(m) !}
$$

Consequently one concludes in fact that

$$
d(X)=\lim _{t \rightarrow \infty} \frac{|X \cap\{1,2, \ldots, t\}|}{t}=1-\sum_{m=1}^{\infty} \frac{1}{k(m) !} .
$$

Now given $n \in \mathbb{N}$, let $a_{n}=(n)$ !. If $m>n$ then $a_{n}$ does not divide $b_{m}-b_{n}$, since $b_{m} \in X_{n+1}$. Thus Corollary 4.3 applies to show that $\rho_{p}$ is one-to-one for each $p \in X$.

We now present two specialized sufficient conditions for right cancellation. Recall that a point $q$ of $\mathbb{N}^{*}$ is a $p$-point of $\mathbb{N}^{*}$ if and only if the intersection of any countable collection of neighbourhoods of $q$ is again a neighbourhood of $q$ (in $\mathbb{N}^{*}$ ). Their existence is known to be independent of the axioms of $Z F C$. (See [5].)

Theorem 4.7. Let $p \in \mathbb{N}^{*}$. If either $p$ is a p-point of $\mathbb{N}^{*}$ or there is an increasing sequence $\left\langle k(n)>_{n=1}^{\infty}\right.$ in $\mathbb{N}$ such that $p \in$ int $_{\mathbb{N}}\left(\bigcap_{n=1}^{\infty} \overline{\mathbb{N} k(n))}\right.$, then $\rho_{p}$ is one-to-one.

Proof. If $p$ is a $p$-point of $\mathbb{N}^{*}$ let $k(n)=n$ for each $n$. Given $n \in \mathbb{N}$, let $q_{n}$ be the natural homomorphism from $\beta \mathbb{N}$ onto $\mathbb{Z}_{k(n)}$. We show that under either hypothesis there is some $A \in p$ such that for all $n \in \mathbb{N}$ and all $r \in \bar{A} \backslash \mathbb{N}, q_{n}(r)=q_{n}(p)$. If $p \in$ int $_{\mathbb{N}^{*}}\left(\bigcap_{n=1}^{\infty} \overline{\mathbb{N} k(n)}\right)$, pick $A \in p$ with $\bar{A} \backslash \mathbb{N} \bigcap_{n=1}^{\infty} \overline{\mathbb{N} k(n)}$. Then for each $n \in \mathbb{N}$ and each $r \in \bar{A} \backslash \mathbb{N}, q_{n}(r)=0=q_{n}(p)$. Assume then that $p$ is a $p$-point of $\mathbb{N}^{*}$. For each $n \in \mathbb{N}$ pick $A_{n} \in p$ such that $q_{n}\left[A_{n}\right]=\left\{q_{n}(p)\right\}$. Pick $A \in p$ such that $A \backslash A_{n}$ is finite for each $n$. If $r \in \bar{A} \backslash \mathbb{N}$, then for each $n, q_{n}(r)=q_{n}(p)$.

By Theorem 4.1 it suffices to let $r, s \in \bigcap_{n=1}^{\infty} \overline{\mathbb{N} n}$ and show that if $r+p=s+p$, then $r=s$. Assume then we have $r, s \in \bigcap_{n=1}^{\infty} \overline{\mathbb{N} n}$ with $r+p=s+p$. For each $n$ let $B_{n}=A \backslash\{1,2, \ldots, n\}$. Then each $B_{n} \in p$ so $r+p \in r+\bar{B}=c l\left(r+B_{n}\right)$ and $s+p \in s+\bar{B}_{n}=c l\left(s+B_{n}\right)$. Since $c l\left(r+B_{n}\right) \cap$ $c l\left(s+B_{n}\right) \neq \varnothing$ we have by Lemma 1.1 that either $c l\left(r+B_{n}\right) \cap\left(s+B_{n}\right) \neq \varnothing$ or $\left(r+B_{n}\right) \cap$ $c l\left(s+B_{n}\right) \neq \varnothing$. Pick $t_{n} \in c l B_{n}$ and $a_{n} \in B_{n}$ such that either $r+t_{n}=s+a_{n}$ or $r+a_{n}=s+t_{n}$. If $t_{n} \in \mathbb{N}$ we conclude that $r=s$. (Assume $t_{n} \in \mathbb{N}$. Pick $m>\max \left\{t_{n}, a_{n}\right\}$ and assume say $r+t_{n}=s+a_{n}$. Then $\mathbb{N} m+t_{n} \in r+t_{n}$ and $\mathbb{N} m+a_{n} \in s+a_{n}$ so $\left(\mathbb{N} m+t_{n}\right) \cap\left(\mathbb{N} m+a_{n}\right) \neq \varnothing$ so $t_{n}=a_{n}$ so $r=s$.) Thus we assume $t_{n} \in \bar{B}_{n} \backslash \mathbb{N}$.

Without loss of generality we have for infinitely many values of $n$ that $r+t_{n}=s+a_{n}$. Pick one such $n$ and pick $m>a_{n}$ such that $r+t_{m}=s+a_{m}$. Then $a_{m} \in B_{m}$ so $a_{m}>a_{n}$. Pick $l$ such that $k(l)>a_{m}$. Now $q_{l}(s)+q_{l}\left(a_{n}\right)=q_{l}\left(s+a_{n}\right)=q_{l}\left(r+t_{n}\right)=q_{l}(r)+q_{l}\left(t_{n}\right)=q_{l}(r)+q_{l}(p)=$ $q_{l}(r)+q_{l}\left(t_{m}\right)=q_{l}\left(r+t_{m}\right)=q_{l}\left(s+a_{m}\right)=q_{l}(s)+q_{l}\left(a_{m}\right)$. Then $q_{t}\left(a_{n}\right)=q_{l}\left(a_{m}\right)$ while $k(l)>a_{m}>a_{n}$ so $q_{1}\left(a_{n}\right)=a_{n}$ and $q_{l}\left(a_{m}\right)=a_{m}$. This contradiction completes the proof.

Note that the following theorem does not include any topological assumptions about $S$. 
Theorem 4.8. Let $(S,+)$ be a cancellative semigroup and let $h: \beta \mathbb{N} \rightarrow S$ be such that $h$ is one-to-one on $\mathbb{N}$ and for all $q \in \beta \mathbb{N}$ and all $r \in \bigcap_{n=1}^{\infty} \mathbb{N} n, h(r+q)=h(r)+h(q)$. For each $n$ let $K(n)=\left\{q \in \mathbb{N}^{*}: h(q)=h(n)\right\}$. If $p \in \mathbb{N}^{*}$ and $\rho_{p}$ is not one-to-one on $\beta \mathbb{N}$, then $p \in c l\left(\bigcup_{n=1}^{\infty} b d y_{\mathrm{N}} \cdot K(n)\right)$.

Proof. By Theorem 4.1, pick $q \neq r$ in $\bigcap_{n=1}^{\infty} \overline{\mathbb{N} n}$ such that $q+p=r+p$. Suppose that $p \notin c l\left(\bigcup_{n=1}^{\infty} b d y_{N^{*}} K(n)\right)$ and pick some $A \in p$ such that $\bar{A} \cap\left(\bigcup_{n=1}^{\infty} b d y_{N^{*}} K(n)\right)=\varnothing$. For each $n \in \mathbb{N}$, let $U_{n}=\bar{A} \cap K(n)$. We show each $U_{n}$ is clopen in $\mathbb{N}^{*}$. Indeed we have $\bar{A} \cap\left(K(n) \backslash\right.$ int $\left._{\mathbb{N}^{*}} K(n)\right)=\varnothing$ so $U_{n}=\bar{A} \cap \operatorname{int}_{N^{*}} K(n)$ so $U(n)$ is open in $\mathbb{N}^{*}$. Also $\bar{A} \cap$ $(c l K(n) \backslash K(n))=\varnothing$ so $U_{n}=\bar{A} \cap \operatorname{cl} K(n)$ so $U_{n}$ is closed in $\mathbb{N}^{*}$.

We claim $p \in \operatorname{cl}\left(\bigcup_{n=1}^{\infty} U_{n}\right)$. Let $B \in p$. Since $q+p=r+p$, we have $(q+\operatorname{cl}(A \cap B)) \cap$ $(r+\operatorname{cl}(A \cap B)) \neq \varnothing$ so without loss of generality, by Lemma 1.1, we can pick $n \in A \cap B$ and $s \in \operatorname{cl}(A \cap B)$ with $q+n=r+s$. Again, since $q, r \in \bigcap_{n=1}^{\infty} \overline{\mathbb{N} n}$ and $q \neq r$ we cannot have $s \in \mathbb{N}$. Since $h(q)+h(p)=h(r)+h(p)$ we have by right cancellation that $h(q)=h(r)$. Thus by left cancellation we get $h(n)=h(s)$. Thus $s \in K(n)$ and hence $s \in U(n) \cap \bar{B}$ as required.

Thus $q+p=r+p$ is in $\left(q+\operatorname{cl}\left(\bigcup_{n=1}^{\infty} U_{n}\right)\right) \cap\left(r+\operatorname{cl}\left(\bigcup_{n=1}^{\infty} U_{n}\right)\right)$. Again by Lemma 1.1 we may without loss of generality pick some $n \in \mathbb{N}$ and some $u \in U_{n}$ and some $v \in \operatorname{cl}\left(\bigcup_{n=1}^{\infty} U_{n}\right)$ with $q+u=r+v$. Now $v \in c l\left(\bigcup_{n=1}^{\infty} U_{n}\right) \subseteq \bar{A}$ and $h(q)+h(u)=h(r)+h(v)$ so $h(v)=h(u)=h(n)$ so $v \in K(n)$ so $v \in U_{n}$. Since $u$ and $v$ are in $U_{n}$ which is open in $\mathbb{N}^{*}$, pick $C \in u$ and $D \in v$ with $\bar{C} \backslash \mathbb{N} \subseteq U_{n}$ and $\bar{D} \backslash \mathbb{N} \subseteq U_{n}$. We may presume $n \notin C \cup D$. (Just delete it if it is.) Now $(q+c l C) \cap(r+c l D) \neq \varnothing$ so again by Lemma 1.1 pick without loss of generality some $k \in C$ and some $w \in c l D$ with $q+k=r+w$. Again we cannot have $w \in \mathbb{N}$ since $q \neq r$ and $q, r \in \bigcap_{m=1}^{\infty} \overline{\mathbb{N} m}$. Thus $w \in U_{n}$. But we have then by left cancellation $h(k)=h(w)$ while $h(w)=h(n)$. But this contradicts the fact that $h$ is one-to-one on $\mathbb{N}$.

The following corollary is of special interest because $b d y_{N^{*}} T$ is a subsemigroup of $(\beta \mathbb{N},+)$ which includes all of the idempotents.

Corollary 4.9. Let $T=\bigcap_{n=1}^{\infty} \overline{\mathbb{N} n}$. Let $p \in \mathbb{N}^{*}$ such that $\rho_{p}$ is not one-to-one on $\beta \mathbb{N}$. Then $p \in c l \bigcup_{n=1}^{\infty}\left(b d y_{N *}(T+n)\right)$, which is a nowhere dense set.

Proof. Let $S=\bigotimes_{n=2}^{\infty} \mathbb{Z}_{n}$ and let $h$ be the natural homomorphism from $\beta \mathbb{N}$ to $S$. It is easy to see that $h$ satisfies the hypotheses of Theorem 5.5. Now $\rho_{n}$ is a homeomorphism of $\mathbb{N}^{*}$ onto $\mathbb{N}^{*}$ so we need only observe that $T+n=K(n)$ in order to conclude that $b d y_{N^{*}}(T+n)=b d y_{N^{*}} K(n)$. Finally we see that $c l \bigcup_{n=1}^{\infty}\left(b d y_{N^{*}}(T+n)\right)$ has empty interior because in $\mathbb{N}^{*}$ the union of countably many nowhere dense sets is again nowhere dense.

\section{Left and right cancellation in $(\beta \mathbb{N},+)$}

We deal here with the interaction of left and right cancellation in $(\beta \mathbb{N},+)$. Recall from Corollary 4.5 that the set of points at which both left and right cancellation holds has dense interior in $\mathbb{N}^{*}$.

Lemma 5.1. Let $p \in \beta \mathbb{N}$. If $\rho_{p}$ is one-to-one on $\mathbb{N}^{*}$, then $\rho_{p}$ is one-to-one on $\beta \mathbb{N}$. 
Proof. Assume $q, r \in \beta \mathbb{N}$ and $q+p=r+p$. Pick $s \in \mathbb{N}^{*}$ such that $\lambda_{s}$ is one-to-one on $\beta \mathbb{N}$. Then $s+q+p=s+r+p$ and $s+q$ and $s+r$ are in $\mathbb{N}^{*}$ so $s+q=s+r$ and hence $q=r$.

We remark that half of the characterization of Section 3 remains valid (trivially) for right cancellation. That is, if $p \in \beta \mathbb{N}+p$, then $\rho_{p}$ is not one-to-one on $\beta \mathbb{N}$. (Indeed if $p=q+p$ then $1+p=1+q+p$.) We do not know if the other half of the characterization is valid.

The following is a lemma for Theorem 5.3, but we feel it is interesting in its own right. By $F S\left(\left\langle x_{n}\right\rangle_{n=m}^{\infty}\right)$ we mean $\left\{\sum_{n \in F} x_{n}: F\right.$ is a finite nonempty subset of $\mathbb{N}$ and $\min F \geqq m\}$.

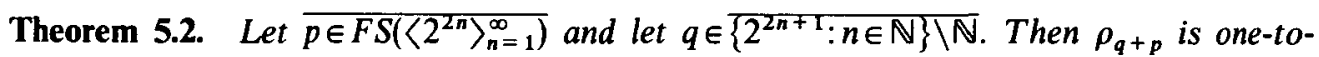
one on $\beta \mathbb{N}$.

Proof. Let $A=\left\{\sum_{n \in F} 2^{n}: \max F \in \mathbb{N} 2+1\right.$ and $\left.F \backslash\{\max F\} \subseteq \mathbb{N} 2\right\}$. We show first that $A \in q+p$. Indeed we simply observe that $F S\left(\left\langle 2^{2 n}\right\rangle_{n=1}^{\infty}\right) \subseteq\{x \in \mathbb{N}: A-x \in q\}$. (For given $x=\sum_{n \in F} 2^{2 n}$ one has $\left\{2^{2 n+1}: n>\max F\right\} \in q$ and $\left\{2^{2 n+1}: n>\max F\right\} \subseteq A-x$.)

Enumerate $A$ in order as $\left\langle b_{n}\right\rangle_{n=1}^{\infty}$. For each $n$ pick $F_{n}$ such that $b_{n}=\sum_{t \in F_{n}} z^{t}$ where $\max F_{n}$ is odd and $F_{n} \backslash\left\{\max F_{n}\right\} \subseteq \mathbb{N} 2$. Let $\sigma(n)=\max F_{n}+1$ and note that if $n<m$, then $\sigma(n) \leqq \sigma(m)$. For $n \in \mathbb{N}$ define $\phi(n)=2^{\sigma(n)}$. We show that for $n<m, \phi(n)$ does not divide $b_{m}-b_{n}$, so that Theorem 4.1 applies. To this end, let $n<m$ be given. Suppose that $b_{m}-b_{n}=a \cdot \phi(n)$. Then $\sum_{t \in F_{m}} z^{t}=a \cdot 2^{\sigma(n)}+\sum_{t \in F_{n}} z^{t}$. By the uniqueness of binary expansions $F_{m}=F_{n} \cup G$ where $a \cdot 2^{\sigma(n)}=\sum_{\mathrm{I} \in G} z^{i}$ (and $\min G \geqq \sigma(n)$ ). But then $\max F_{n} \in F_{m}$ and $\max F_{n}<\max F_{m}$ so $\max F_{n}$ is even, a contradiction.

Theorem 5.3. There exists some $p \in \mathbb{N}^{*}$ such that $\rho_{p}$ is one-to-one on $\beta \mathbb{N}$ but $\lambda_{p}$ is not one-to-one on $\beta \mathbb{N}$.

Proof. Consider $M=\bigcap_{m=1}^{\infty} \overline{F S\left(\left\langle 2^{2 n}\right\rangle_{n=m}^{\infty}\right)}$. It is well known and easy to see that $M$ is a subsemigroup of $\beta \mathbb{N}$ which is in fact isomorphic to $\bigcap_{m=1}^{\infty} \overline{\mathbb{N} 2^{m}}=\bigcap_{m=1}^{\infty} \overline{F S\left(\left\langle 2^{n}\right\rangle_{n=m}^{\infty}\right)}$. The smallest ideal of $\bigcap_{m=1}^{\infty} \overline{\mathbb{N} 2^{m}}$ is contained in $K(\beta \mathbb{N},+) \cap \bigcap_{m=1}^{\infty} \overline{\mathbb{N} 2^{m}}$ (which is a proper subset of $\bigcap_{m=1}^{\infty} \overline{\mathbb{N} 2^{m}}$ ). Consequently, the smallest ideal of $M$ is a proper subset of $M$ and hence we may pick a point $p \in M$ such that $\lambda_{p}$ is not one-to-one on $M$, and hence not on $\beta \mathbb{N}$. Pick $q \in\left\{2^{2 n+1}: n \in \mathbb{N}\right\} \backslash \mathbb{N}$.

By Theorem $5.2 \rho_{q+p}$ is one-to-one on $\beta \mathbb{N}$. On the other hand, given $r \neq s$ such that $p+r=p+s$, one has $(q+p)+r=(q+p)+s$.

Theorem 5.4. There exists some $p \in \mathbb{N}^{*}$ such that $\lambda_{p}$ is one-to-one on $\beta \mathbb{N}$ but $\rho_{p}$ is not one-to-one on $\beta \mathbb{N}$.

Proof. Again consider $M=\bigcap_{m=1}^{\infty} \overline{F S\left(\left\langle 2^{2 n}\right\rangle_{n=m}^{\infty}\right)}$. Again utilizing the fact that $M$ is isomorphic to $\bigcap_{m=1}^{\infty} \overline{\mathbb{N} 2^{m}}$ we get that there is some idempotent $r \in M$ such that $r$ is not an element of the smallest ideal of $M$. Then by [1, Lemma 3.2] we may pick an idempotent $p \neq r$ in $M$ with $p=p+r=r+p$. Pick any $q \in\left\{2^{2 n+1}: n \in \mathbb{N}\right\} \backslash \mathbb{N}$ and consider $p+q$. Now $r+p+q=p+p+q=p+q$ so $\rho_{p+q}$ is not one-to-one. We show that $p+q \notin p+$ $q+\beta \mathbb{N}$ (so by Theorem $2.1 \lambda_{p+q}$ is one-to-one on $\beta \mathbb{N}$ ). 
Let $A=\left\{\sum_{n \in F} 2^{n}: \min F\right.$ is odd and $\left.F \backslash\{\min F\} \subseteq \mathbb{N} 2\right\}$. Then as before we easily see that $A \in p+q$. Now suppose $p+q=p+q+s$ for some $s \in \beta \mathbb{N}$. Then $A \in p+q+s$ so pick $x \in \mathbb{N}$ such that $A-x \in p+q$. Pick $F$ such that $x=\sum_{n \in F} 2^{n}$. Let $t=\max F$. Then $\mathbb{N} 2^{i+1} \in p$ and $\mathbb{N} 2^{t+1} \in q$ so $\mathbb{N} 2^{t+1} \in p+q$. Pick $y \in A \cap(A-x) \cap \mathbb{N} 2^{t+1}$. Pick $G$ such that $y=\sum_{n \in G} 2^{n}$. Then $\min G$ is odd so $(F \cup G) \backslash \min (F \cup G) \nsubseteq \mathbb{N} 2$ so $y+x \notin A$, a contradiction.

We conclude by lengthening the list of characterizations of left cancellation in $(\beta \mathbb{N},+)$.

Theorem 5.5. Let $p \in \mathbb{N}^{*}$. The following statements are equivalent.

(a) $\lambda_{p}$ is one-to-one on $\beta \mathbb{N}$;

(b) $\lambda_{p}$ is one-to-one on $\bigcap_{n=1}^{\infty} c l(\mathbb{N m})$;

(c) there exist a first countable topological group $(G,+)$ and a continuous homomorphism $h: \beta \mathbb{N} \rightarrow G$ such that $\lambda_{p}$ is one-to-one on $\operatorname{ker}(h)$;

(d) $p+\mathbb{N}^{*}$ is not separable;

(e) there exists $A$ in $p$ such that for each $k \in \mathbb{N}, A-k \notin p$.

Proof. The equivalence of (a), (b), and (c) is established in a fashion nearly identical to the proof of Theorem 4.1 (one uses however Corollary 4.5 to obtain $s \in \mathbb{N}^{*}$ with $\left\{x_{n}: n \in \mathbb{N}\right\} \in s$ and with $\rho_{s}$ one-to-one.)

We now show that (a) and (d) are equivalent. To see that (a) implies (d) assume $\lambda_{p}$ is one-to-one. Then $\lambda_{p}$ is a homeomorphism from $\mathbb{N}^{*}$ onto $p+N^{*}$. Since $\mathbb{N}^{*}$ is not separable (see [8]) neither is $p+\mathbb{N}^{*}$. To see that (d) implies (a) suppose that $\lambda_{p}$ is not one-to-one. Then by Theorem 2.1 we have $p \in p+\mathbb{N}^{*}$ and hence $p+\mathbb{N} \subseteq p+\mathbb{N}^{*}+\mathbb{N} \subseteq p+\mathbb{N}^{*}$ so that $\operatorname{cl}(p+\mathbb{N})=p+\mathbb{N}^{*}$.

The equivalence of (a) and (e) follows from Theorem 2.1 and Lemma 3.5.

\section{REFERENCES}

1. J. Baker, N. Hindman, and J. Pym, Elements of finite order in Stone-Čech compactifications, Proc. Edinburgh Math. Soc. 36, (1992), 49-54.

2. V. Bergelson and N. Hindman, Nonmetrizable topological dynamics and Ramsey Theory, Trans. Amer. Math. Soc. 320 (1990), 293-320.

3. J. Berglund, H. Junghenn, and P. Milnes, Analysis on Semigroups (Wiley, New York, 1989).

4. A. Blass and N. Hindman, Sums of ultrafilters and the Rudin-Keisler and Rudin-Frolik orders, in General Topology and Applications (R. Shortt ed., Lectures Notes in Pure and Applied Math. 123, 1990), 59-70.

5. W. Comfort, Ultrafilters-some old and some new results, Bull. Amer. Math. Soc. 83 (1977), 417-455.

6. W. Comfort and S. Negrepontis, The theory of ultrafilters (Springer-Verlag, Berlin, 1974).

7. R. Elus, Lectures on topological dynamics (Benjamin, New York, 1969).

8. L. Gillman and M. Jerison, Rings of continuous functions (Van Nostrand, Princeton, 1960).

9. R. Graham, B. Rothschild, and J. Spencer, Ramsey Theory (Wiley, New York, 1990). 
10. N. Hindman, Minimal ideals and cancellation in $\beta \mathbb{N}$, Semigroup Forum 25 (1982), 291-310.

11. N. Hindman, Sums equal to products in $\beta \mathbb{N}$, Semigroup Forum 21 (1980), 221-255.

12. N. Hindman, The ideal structure of the space of $\kappa$-uniform ultrafilters on a discrete semigroup, Rocky Mountain J. Math. 16 (1986), 685-701.

13. N. Hindman, The semigroup $\beta \mathbb{N}$ and its applications to number theory, in The Analytical and Topological Theory of Semigroups-Trends and Developments (K. Hofmann, J. Lawson, and J. Pym eds., de Gruyer Expositions in Math. 1, 1990), 347-360.

14. P. Milnes, Compactifications of topological semigroups, J. Australian Math. Soc. 15 (1973), 488-503.

15. F. Ramsey, On a problem of formal logic, Proc. London Math. Soc. 30 (1930), 264-286.

16. D. Strauss, A proof that $\mathbb{N}^{*}$ does not contain an algebraic and topological copy of $\beta \mathbb{N}, J$. London Math. Soc. (2) 46 (1992), 463-470.

17. D. Strauss, Semigroup structures on $\beta \mathbb{N}$, Semigroup Forum 44 (1992), 238-244.

Department of Mathematics

HOWARD UNIVERSITY

WASHINGTON D.C. 20059

U.S.A.
Department of Pure Mathematics UNIVERSITY OF HULL

CotTingham Road

Hull HU6 7RX 